\title{
Epidemiology of the lymphatic-dwelling filarioid nematode Rumenfilaria andersoni in free-ranging moose (Alces alces) and other cervids of North America
}

Caroline M. Grunenwald', Michelle Carstensen², Erik Hildebrand², Jacob Elam, Sauli Laaksonen³, Antti Oksanen ${ }^{4}$ and Richard W. Gerhold ${ }^{5^{*}}$

\begin{abstract}
Background: Moose (Alces alces) are a culturally and economically valued species in Minnesota, where the northeast population has decreased by $60 \%$ since 2006 . The cause of the decline is currently unclear; however, parasites, predation, and climate change have all been implicated. Nematode parasites are important pathogens in North American moose, potentially causing severe disease and mortality. Recent spread of Rumenfilaria andersoni, a filarioid nematode of moose, has been documented in Finnish cervids; however, little is known about the epidemiology of this parasite in North America.

Methods: To investigate the prevalence and distribution of $R$. andersoni, 584 blood samples were collected from live-captured and dead animals and screened microscopically for the presence of microfilariae using a modified Knott's test. Microfilariae were identified based on morphological characteristics. A subset of Knott's-positive animals was subjected to polymerase chain reaction (PCR) with filarioid-specific primers targeting the first internal transcribed spacer region (ITS-1) of the rRNA gene cluster.

Results: Rumenfilaria microfilariae were present in $20.5 \%$ of Minnesota moose $(n=352)$, with slight fluctuations observed over four years. Minnesota white-tailed deer (Odocoileus virginianus) $(n=2)$ and moose $(n=44)$ from Alaska, Montana, Washington, Maine, and New Hampshire also harbored $R$. andersoni, suggesting this parasite occurs widely throughout North American moose herds, and white-tailed deer can serve as a patent host. Sequence analysis of cervid blood (moose, $n=15$; white-tailed deer, $n=1$ ) confirmed the identity of $R$. andersoni and revealed the existence of two distinct clades. Genetic comparisons of $R$. andersoni isolates from North America and semi-domesticated Finnish reindeer found the two groups to be closely related, supporting previous hypotheses that $R$. andersoni was recently introduced into Finland by the importation of deer from the United States.

Conclusions: To the best of our knowledge these observations represent the first report of $R$. andersoni within the contiguous United States and reveal this nematode as a common parasite of North American moose and white-tailed deer. Although the implications of $R$. andersoni infection on moose health is unclear, increased awareness of this parasite will help prevent unintentional introduction of $R$. andersoni into naïve populations via the translocation of wild and captive cervids.
\end{abstract}

Keywords: Rumenfilaria andersoni, Cervids, Lymphatic filariasis, Bioinvasion, Parasite translocation, Moose (Alces alces), White-tailed deer (Odocoileus virginianus)

\footnotetext{
* Correspondence: rgerhold@utk.edu

${ }^{5}$ Department of Biomedical and Diagnostic Sciences, College of Veterinary

Medicine, University of Tennessee, 2407 River Drive, Knoxville, TN 37996, USA

Full list of author information is available at the end of the article
} 


\section{Background}

Moose (Alces alces) have long been a culturally and economically valued species in North America; however, some North American moose populations have exhibited a serious decline in population [1-3]. Nowhere has this decline been more dramatic than in Minnesota, where the estimated number of free-ranging moose in the northeastern region has decreased by $60 \%$, from 8840 animals in 2006 to 3450 in 2015 [4]. Disease, parasites, predation, and climate change have all been implicated as factors related to the decline [5-9]. To prevent further loss of this species and natural resource, a better understanding of the Minnesota moose herd's overall health, as well as an understanding of the potential drivers of mortality, is urgently needed.

Nematode parasites, particularly species of lungworms and filarioids, are important pathogens in moose and are known to cause morbidity and mortality in freeranging populations [6,10-12]. Rumenfilaria andersoni is a lymphatic-dwelling filarioid nematode associated with moose and caribou (Rangifer tarandus). The species belongs to the family Onchocercidae, which is a group of filarioid nematodes transmitted by hematophagous arthropods. Although the exact details of the $R$. andersoni lifecycle have yet to be elucidated, all adult filarioids of the family Onchocercidae produce larval stages called microfilariae that reside in the circulatory system of the definitive cervid host. When an arthropod intermediate host (vector) obtains a blood meal and ingests the microfilariae, the microfilariae unsheathe, penetrate the vector's gut wall, and develop to an infectious larval stage within the vector's hemocoel. Extrinsic development is completed when infectious larvae are inoculated into a definitive host by the vector during a subsequent blood meal.

Originally described in moose from Ontario [13] and more recently in Alaska [14] and Finland [15, 16], the exact geographical distribution and vector identity for $R$. andersoni are unknown. Although the pathological impact of this filarioid nematode on cervid health remains unclear, recent studies documenting the rapid expansion of $R$. andersoni in Finnish semi-domesticated reindeer ( $R$. tarandus tarandus) describe inflammation of ruminal lymphatic vessels and high microfilariae load within the bloodstream $[15,16]$, both of which are predicted to have a negative impact on overall cervid health [17-19]. These results suggest $R$. andersoni infections may have important health implications for cervids, including moose.

The main objective of this study was to investigate the eco-epidemiology of $R$. andersoni in Minnesota moose compared to other North American herds. To accomplish this, we explored the basic epidemiology of this parasite, including the identification of other patent host species, geographical distribution, prevalence of infection, and host demographics by collecting parasitological samples across spatial and temporal scales. We also performed the first genetic characterization and comparison of $R$. andersoni isolates to provide a basis for future population genetics studies. These data will contribute to a greater understanding of $R$. andersoni biology and provide baseline epidemiological data for future reference and research.

\section{Methods}

Sampling of hosts and detection of microfilariae

To estimate the prevalence of $R$. andersoni within the Minnesota moose population, 352 blood samples were collected between 2012 and 2015. Blood was collected from either hunter-killed animals, opportunistic mortalities, or live-captured animals and placed into 5-ml EDTA blood tubes. A limited number of blood samples were also obtained from hunter-harvested wild elk (Cervus canadensis) $(n=14)$ and live-captured and hunter-harvested free-ranging white-tailed deer (Odocoileus virginianus) $(n=36)$ in Minnesota. Additional blood samples were donated by various state wildlife agencies from live-captured and hunter-harvested animals, including 12 caribou and 27 moose from Alaska; 14, 73, and 16 moose from Maine, Montana, and New Hampshire; and 16 moose, 1 white-tailed deer, and 3 mule deer (Odocoileus hemionus) from Washington. Whenever possible, the age, sex, and geographical location of the animal was noted. Animals were classified as calves ( $<1$ year of age), yearlings ( 1 to $<2$ years of age), or adults ( $\geq 2$ years old). Blood samples $(1 \mathrm{ml} /$ animal) were refrigerated and shipped to the Molecular Parasitology Laboratory at the University of Tennessee College of Veterinary Medicine to identify the presence of $R$. andersoni microfilariae (RMF) using a modified Knott's test and bright-light microscopy [20]. Microfilariae were identified based on comparative morphological features [15, 20, 21] (Fig. 1d).

\section{Prevalence estimates and statistical analyses}

Prevalence of $R$. andersoni within cervid populations was estimated for each cervid species based on the modified Knott's test results. Blood samples were categorized as RMF positive based on the presence of microfilariae with morphological features consistent with $R$. andersoni identified via the modified Knott's test described above. Animals with no microfilariae present or microfilariae morphologically distinct from $R$. andersoni were categorized as RMF negative. To determine if $R$. andersoni prevalence differed between geographical locations, a Pearson's $\chi^{2}$ test or Fisher's exact test was used with Bonferroni correction $(P \leq 0.012)$. The Minnesota moose population was further analyzed by comparing prevalence between age class, sex, and sample year using the Pearson's $\chi^{2}$ test or Fisher's exact test with Bonferroni correction $(P \leq 0.012)$. Statistical analyses were performed with 

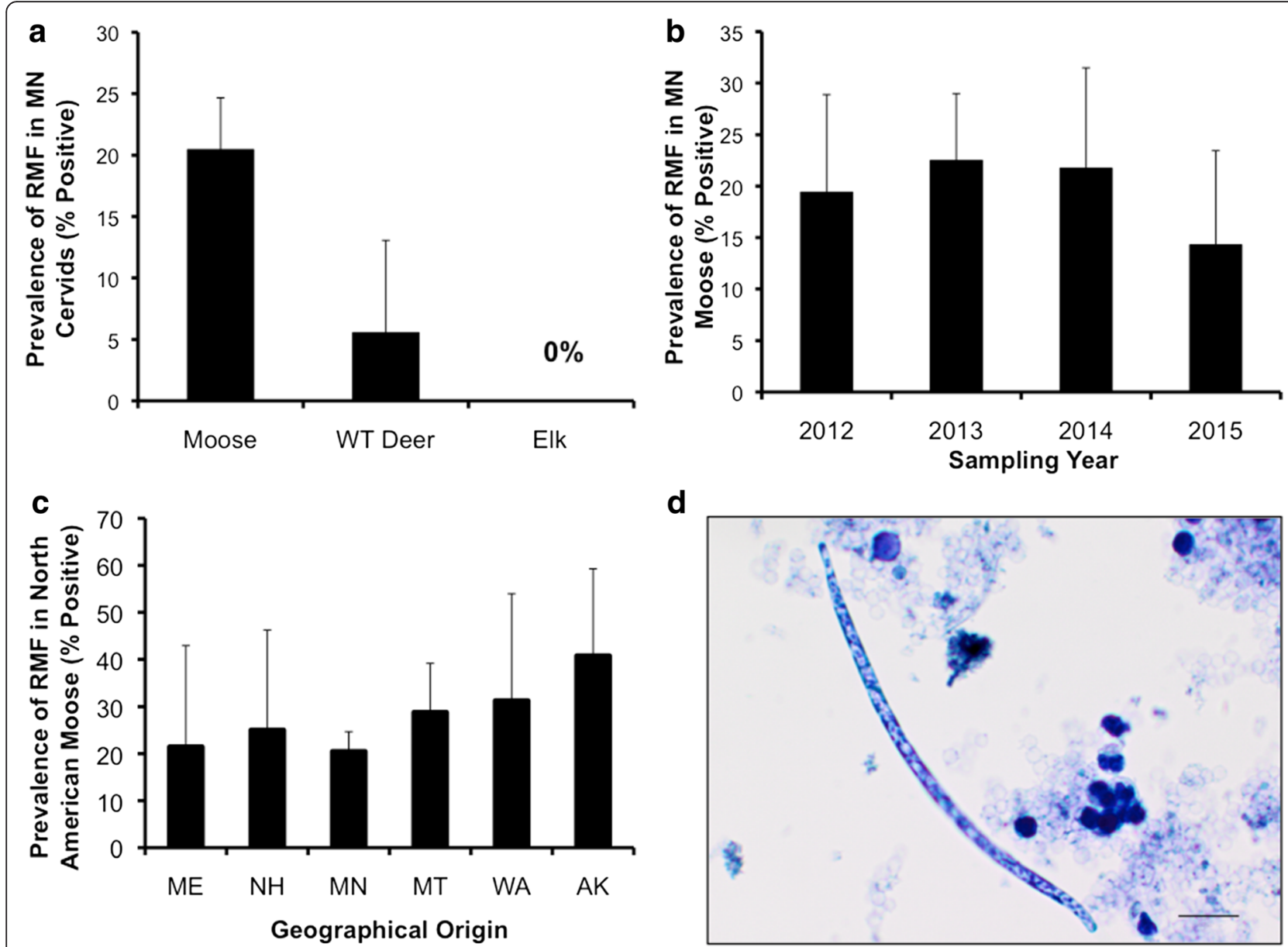

d

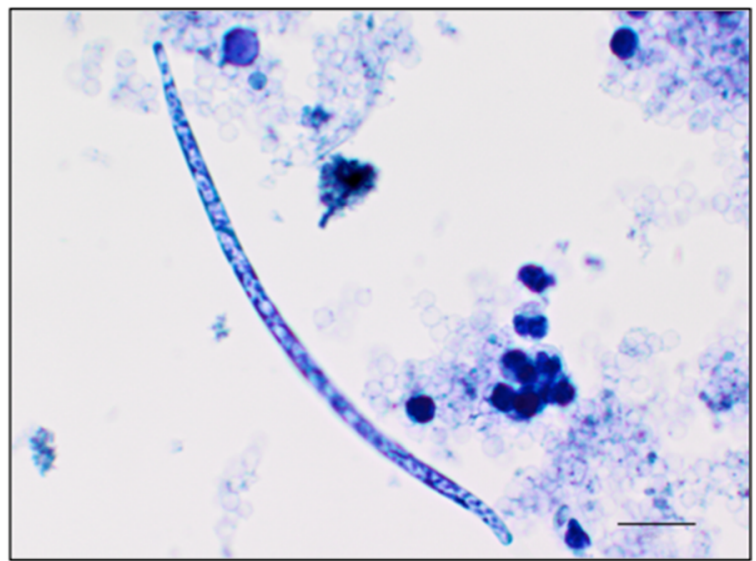

Fig. 1 Prevalence of $R$. andersoni microfilariae (RMF) in blood samples drawn from free-ranging cervids. Prevalence is defined as the percentage of samples that tested RMF-positive using a modified Knott's test. Error bars represent $95 \%$ confidence intervals. a Prevalence of RMF in three species of Minnesota cervids (moose, $n=352$; elk, $n=14$; white-tailed (WT) deer, $n=36$ ). Blood samples were collected over a four-year period for moose and a two-year period for elk and deer. Fisher's exact test; $P=0.013$. b Comparison of RMF prevalence in Minnesota moose over time $(2012, n=67 ; 2013, n=160 ; 2014, n=69 ; 2015, n=56)$. Fisher's exact test; $P=0.607$. c Comparison of RMF prevalence in moose from several U.S. states (MN, $n=352 ; \mathrm{NH}, n=16 ; \mathrm{ME}, n=14 ; \mathrm{MT}, n=73 ; \mathrm{WA}, n=16 ; \mathrm{AK}, n=27)$. Fisher's exact test; $P=0.013$. $\mathbf{d}$ Image of RMF from Minnesota moose blood. Sample was stained with methylene blue and viewed with a bright light microscope at 200x magnification. Scale-bar: $20 \mu \mathrm{m}$

SPSS software version 23.0 (IBM Corporation, Armonk, NY, USA).

\section{Molecular confirmation of microfilariae as $R$. andersoni}

To confirm the identity of the RMF, as well as investigate intra-species genetic variation, 30 Knott's-positive blood samples containing microfilariae that were morphologically consistent with $R$. andersoni were selected for molecular analysis. DNA was extracted using the ZR Fecal DNA Kit (Zymogen, Irvine, CA, USA) according to the manufacturer's instructions. Nuclease-free water served as the DNA extraction control. RMF DNA was amplified using a previously described semi-nested PCR protocol targeting the first internal transcribed spacer region (ITS-1) of the rRNA gene cluster [22]. The primers FL1-F (5' -TTC CGT AGG TGA ACC TGC-3') and FL2-R (5' ${ }^{\prime}$ ATA TGC TTA AAT TCA GCG GG-3') were used in the primary $\mathrm{PCR}$ reaction; the primers FL1-F and Di58S660 (5'-ACC CTC AAC CAG ACG TAC-3') were used in the secondary PCR reaction [22]. DNA from a morphologically-identified adult $R$. andersoni nematode (RA-F3) isolated from a Finnish reindeer and nuclease-free water served as the positive and negative PCR controls, respectively. The PCR products were separated by agarose gel electrophoresis and viewed under UV light. PCR amplicons around $600 \mathrm{bp}$ were excised using a clean razor blade and the PCR product purified using the QIAquick Gel Extraction Kit (Qiagen, Valencia, CA, USA). The ITS-1 PCR product was then cloned into a pGEM-T Easy vector (Promega, Madison, WI, USA) and transformed into competent DH5 $\alpha$ Escherichia coli cells (Invitrogen, Grand Island, NY, USA) via a 40-second heat shock at $42{ }^{\circ} \mathrm{C}$. Transformed cells were cultivated in S.O.C. medium (Life Technologies, Grand 
Island, NY, USA) for $1.5 \mathrm{~h}$ at $30{ }^{\circ} \mathrm{C}$ with shaking. The transformed cells $(100 \mu \mathrm{l})$ were then plated on Luria broth agar plates containing $1 \mu \mathrm{g} / \mathrm{ml}$ carbenicillin and $100 \mu \mathrm{l}$ ChromoMax IPTG/X-gal solution (Thermo Fisher Scientific, Waltham, MA, USA) as a top dressing. Cultures were incubated $24-48 \mathrm{~h}$ at $30{ }^{\circ} \mathrm{C}$; single, white colonies were selected with a sterile toothpick and grown overnight in $5 \mathrm{ml}$ Luria broth with $1 \mu \mathrm{g} / \mathrm{ml}$ carbenicillin. Cultures were centrifuged and the supernatant removed. Plasmids were purified from the remaining cell pellet using the Qiagen Spin Miniprep Plasmid Kit (Qiagen) following the manufacturer's instructions. To confirm the presence of the filarioid ITS-1 PCR product insert, the plasmids were digested with EcoR1 restriction enzyme (Thermo Fisher Scientific) and examined via gel electrophoresis for multiple bands. Plasmids containing an insert of approximately $600 \mathrm{bp}$ were sequenced at the University of Tennessee's Genomics Core (Knoxville, TN, USA).

\section{Phylogenetic analysis of $R$. andersoni sequences}

All 18 S and ITS-1 consensus sequence chromatograms were trimmed and edited by hand using Sequencher 5.3 (Gene Codes Corporation, Ann Arbor, MI, USA). Edited sequences were compared against the few known sequences for filarioid nematodes from cervid hosts in the NCBI GenBank database. Genetic data was also compared with sequences obtained from adult reference nematodes (Table 1), which had been identified morphologically and subjected to DNA extraction and PCR amplification in our laboratory, as described above. Alignment and construction of neighbor-joining trees of ITS-1 and 18S filarioid worm sequences was performed using MEGA 6.0 [23]. All consensus sequences were deposited in the GenBank database under accession numbers KT020850, KT031392-KT031393; KT873719-KT873733; KT878970KT878979l; and KU757075-KU757077 (see Tables 1 and 3 for details).

\section{Results}

Prevalence of $R$. andersoni in free-ranging cervids of Minnesota and other U.S. states

During 2012-2015, RMF occurred in 20.5 \% (95 \% confidence interval [CI]: 16.3-24.7 \%; $n=352)$ of Minnesota moose (Table 2). Presence of RMF among the Minnesota moose was independent of host gender $\left(\chi^{2}=3.879, d f=1\right.$, $P=0.049)$ and age (Fisher's exact test, $P=0.104$ ). RMF were also detected in Minnesota white-tailed deer, with an overall prevalence of $5.6 \%$ (95 \% CI: $0-13.1 \% ; n=36$ ), but no RMF were detected in the Minnesota elk samples $(0 \%, n=14)$ (Fig. 1a). Over the 4-year sampling period, RMF prevalence in the Minnesota moose varied slightly, ranging from $22.5 \%$ (95\% CI: $16.0-29.0 \% ; n=160)$ in 2013 to $14.3 \%$ (95\% CI: $5.1-23.5 \% ; n=56$ ) in 2015, but differences among sampling years were not significant (Fisher's exact test, $P=0.607$ ) (Fig. 1b).

RMF were also observed in all other surveyed moose populations, including those in Maine at $21.4 \%$ (95\% CI: $0-42.9 \% ; n=14)$, New Hampshire at $25.0 \%$ (95\% CI: $3.8-46.2 \% ; n=16)$, Montana at $28.8 \%(95 \%$ CI: 18.4-39.2 \%; $n=73)$, Washington at $31.3 \%$ (95 \% CI: 8.6-54 \%; $n=16)$, and Alaska at $40.7 \%$ (95 \% CI: $22.2-$ $59.2 \% ; n=27$ ) (Fig. 1c). No statistically significant differences were detected among all populations, including Minnesota (Fisher's exact test, $P=0.013$ ). Moreover, we failed to observe RMF in any of the Alaskan caribou blood samples or in mule or white-tailed deer from Washington.

\section{Genetic comparison of $R$. andersoni isolates}

To confirm the identity of the RMF observed in the Knott's tests and to investigate the intra-species genetic variation of $R$. andersoni, partial filarial ITS-1 sequences from RMF-positive blood samples of 15 North American moose and one white-tailed deer were sequenced (Table 3). Moose isolates varied in geographical origin, with five

Table 1 Reference nematodes used in molecular analysis of blood samples obtained from cervids

\begin{tabular}{|c|c|c|c|c|c|}
\hline Isolate & Species & Geographical origin & Host species & DNA target & GenBank accession no. \\
\hline RA-F124 & Rumenfilaria andersoni & Finland & Rangifer tarandus & 185 & KT878978 \\
\hline RA-F113 & Rumenfilaria andersoni & Finland & Rangifer tarandus & 185 & KT878977 \\
\hline RA-F128 & Rumenfilaria andersoni & Finland & Rangifer tarandus & $18 \mathrm{~S}$ & KT878979 \\
\hline RA-F3 & Rumenfilaria andersoni & Finland & Rangifer tarandus & ITS-1 & KT873731 \\
\hline ES-WY11 & Elaeophora schneideri & Wyoming, USA & Alces alces & $18 \mathrm{~S}$ & KT031392 \\
\hline ES-WY50 & Elaeophora schneideri & Wyoming, USA & Alces alces & ITS-1 & KT873732 \\
\hline ES-CA1 & Elaeophora schneideri & California, USA & Rusa unicolor & 185 & КT020850 \\
\hline OC-AK1 & Onchocerca cervipedis & Alaska, USA & Alces alces & 185 & KT031393 \\
\hline SY-AK1 & Setaria yehi & Alaska, USA & Alces alces & 185 & KT878970 \\
\hline SY-GA3 & Setaria yehi & Georgia, USA & Odocoileus virginianus & ITS-1; $18 \mathrm{~S}$ & KU757075; KT878972 \\
\hline
\end{tabular}

Adult nematodes were identified based on morphological characteristics. Geographial origin and host species refer to the place and host from which the nematode was isolated. DNA target refers to the targeted gene sequence (18S rRNA or ITS-1) that was amplified 
Table 2 Demographics of Minnesota moose sampled for nematode microfilariae prevalence from 2012 to 2015

\begin{tabular}{|c|c|c|c|c|c|c|c|c|c|}
\hline \multirow{2}{*}{ Year } & \multirow[t]{2}{*}{ Season } & \multicolumn{3}{|l|}{ Sex } & \multicolumn{4}{|l|}{ Age } & \multirow[t]{2}{*}{ \% RMF-positive } \\
\hline & & $M$ & $\mathrm{~F}$ & Unknown & $<1$ year & $1-<2$ years & $\geq 2$ years & Unknown & \\
\hline 2012 & Autumn & 62 & 4 & 1 & 1 & 4 & 59 & 3 & $19.4(13 / 67)$ \\
\hline \multirow[t]{4}{*}{2013} & Winter & 28 & 103 & 2 & 1 & 9 & 121 & 2 & $22.6(30 / 133)$ \\
\hline & Spring & 4 & 10 & 1 & 1 & 3 & 10 & 1 & $26.7(4 / 15)$ \\
\hline & Summer & 1 & 6 & 0 & 1 & 1 & 5 & 0 & $14.3(1 / 7)$ \\
\hline & Autumn & 1 & 4 & 0 & 1 & 0 & 4 & 0 & $20.0(1 / 5)$ \\
\hline \multirow[t]{4}{*}{2014} & Winter & 14 & 42 & 1 & 0 & 4 & 50 & 3 & $21.1(12 / 57)$ \\
\hline & Spring & 0 & 3 & 0 & 1 & 1 & 1 & 0 & $66.7(2 / 3)$ \\
\hline & Summer & 1 & 3 & 0 & 1 & 0 & 3 & 0 & $25.0(1 / 4)$ \\
\hline & Autumn & 3 & 2 & 0 & 0 & 2 & 3 & 0 & $0(0 / 5)$ \\
\hline \multirow[t]{3}{*}{2015} & Winter & 14 & 37 & 0 & 0 & 2 & 48 & 1 & $13.7(7 / 51)$ \\
\hline & Spring & 1 & 2 & 0 & 0 & 1 & 1 & 1 & $0(0 / 3)$ \\
\hline & Summer & 1 & 1 & 0 & 0 & 0 & 2 & 0 & $50.0(1 / 2)$ \\
\hline
\end{tabular}

Seasons are defined as winter (December-February), spring (March-May), summer (June-August), and autumn (September-November) Abbreviations: $F$ female, $M$ male, RMF Rumenfilaria andersoni microfilariae

from Montana, eight from Minnesota, and two from Maine. Attempts to amplify additional isolates were not successful. Sequences obtained from a morphologically confirmed $R$. andersoni adult nematode (RA-F3) isolated from a Finnish reindeer served as the standard.

Overall, the RMF ITS-1 sequences were AT-rich, with multiple sections of repeats with variations in length between isolates. Phylogenetic analysis revealed that all of the ITS-1 sequences obtained from the RMF-positive blood clustered with the RA-F3 standard, branching

Table 3 Rumenfilaria andersoni ITS-1 target DNA sequences amplified from cervid blood. Unless otherwise indicated, isolates were obtained from moose (Alces alces)

\begin{tabular}{lll}
\hline Isolate & Geographical origin (USA) & GenBank accession no. \\
\hline RA-MT4 & Montana & KT873721 \\
RA-MT8 & Montana & KT873724 \\
RA-MT31 & Montana & KT873720 \\
RA-MT43 & Montana & KT873722 \\
RA-MT44 & Montana & KT873723 \\
RA-MN1 & Minnesota & KU757076 \\
RA-MN2 & Minnesota & KT873733 \\
RA-MN3 & Minnesota & KT873727 \\
RA-MN4 & Minnesota & KT873728 \\
RA-MN5 & Minnesota & KT873729 \\
RA-MN6 & Minnesota & KT873730 \\
RA-MN7 & Minnesota & KU757077 \\
RA-MN9 & Minnesota & KT873719 \\
RA-ME1 & Maine & KT873726 \\
RA-ME2 & Maine & KT873725 \\
\hline
\end{tabular}

${ }^{\mathrm{a}}$ Host was a white-tailed deer (Odocoileus virginianus) into two distinct $R$. andersoni lineages (Fig. 2). The Minnesota isolates had representatives clustering into both clades, which we simply denoted as Clades A and B. All Montana isolates and six Minnesota isolates clustered into Clade A, which also contained the RA-F3 standard. Clade B contained both Maine isolates and two Minnesota isolates. Isolates of Clades A and B had a mean difference of $0.038(\mathrm{SE}=0.007)$ base substitutions per base pair. Within clades, isolates had a mean difference of 0.015 (Clade A, SE $=0.003$; Clade B, $\mathrm{SE}=0.004$ ) base substitutions per base pair for both Clades A and B.

\section{Identification of the filarioid Setaria yehi in Minnesota moose}

In addition to RMF, another morphologically distinct group of microfilariae was observed in $1.4 \%$ (5/352) of Minnesota moose. These microfilariae were characterized by blunt, rounded heads and long, thinly tapered tails, measuring between 285 and $315 \mu \mathrm{m}$ long and 5-7.5 $\mu \mathrm{m}$ wide (Fig. 3a). Dual infection with RMF and non-RMF microfilariae was observed in a single Minnesota moose. To identify this unknown filarioid, we attempted to sequence a portion of the 18S rRNA gene using nematode-wide primers from the five positive Minnesota moose blood samples [24]. Only one blood sample was successfully PCR amplified. A comparison of 796 base pairs from the unknown filarioid and $18 \mathrm{~S}$ sequences from our reference nematodes (Table 1) revealed the unknown filarioid most closely resembled Setaria yehi (Spirurida: Onchocercidae) [25] (Fig. 3b).

\section{Discussion}

Prior to the conclusion of this study, little was known about the occurrence of $R$. andersoni in cervid hosts. Knowledge about the distribution of $R$. andersoni was 


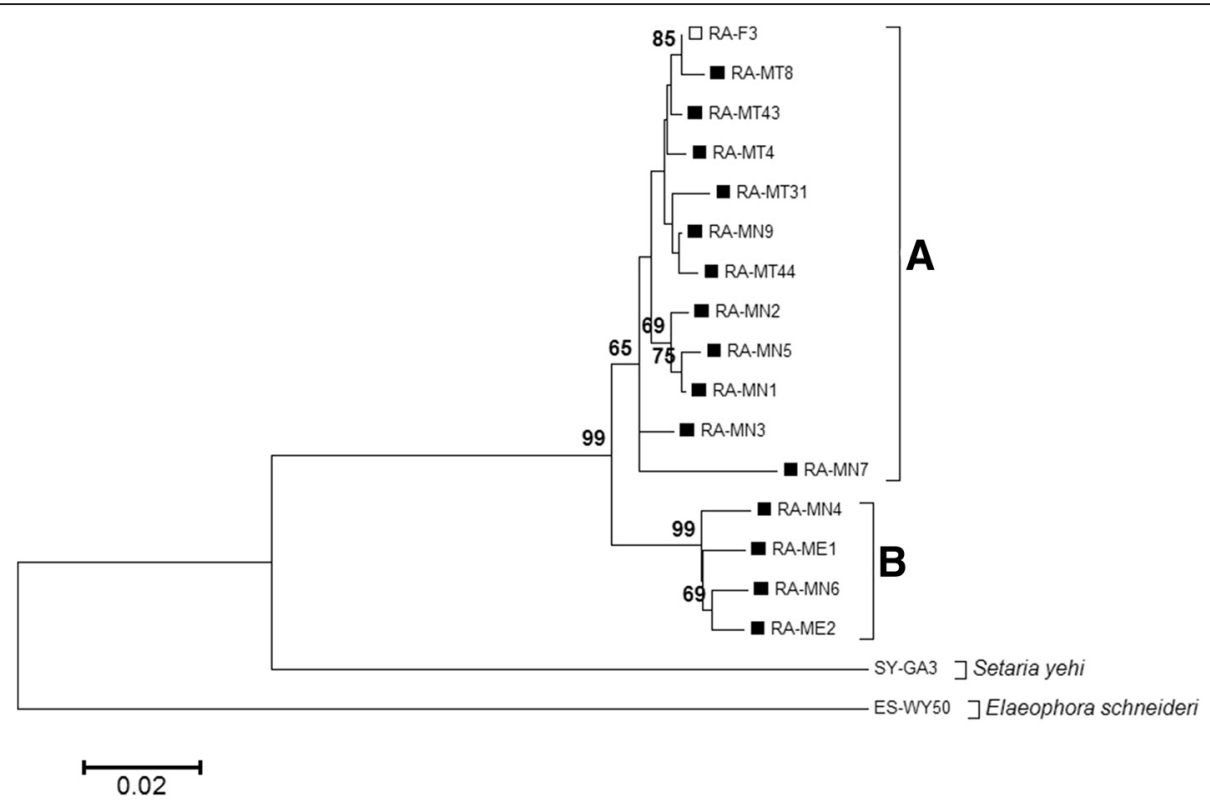

Fig. 2 Phylogenetic analysis of ITS-1 sequences obtained from RMF-positive blood samples from cervid hosts. Sequences of 609 base pairs were aligned using ClustalW, and the evolutionary history was inferred using the Neighbor-Joining method. Evolutionary distances were computed using the Kimura 2-parameter method. The tree is drawn to scale. Bootstrap values ( $\times 1000)$ greater than $50 \%$ are shown above the branches. RMF isolates are marked with solid boxes; ITS-1 Clades A and B are labelled. RA-F3 (Rumenfilaria andersoni; open box), Setaria yehi, and Elaeophora schneideri serve as reference standards. GenBank accession numbers for all isolates are listed in Tables 1 and 3

limited to the original description of this nematode in moose of Ontario [13] and was only recently expanded to include Alaska [14] and Finland [15]. Our survey of moose herds for $R$. andersoni suggests the geographical range of this filarioid nematode is much more extensive than was previously appreciated. RMF were detected in all of the wild moose herds we sampled, including herds geographically isolated from one another (Fig. 1c). These results imply that $R$. andersoni nematodes are widely distributed throughout the North American moose range and support previous studies suggesting moose might serve as the main reservoir host of $R$. andersoni $[13,16]$. Interestingly, Alaskan moose had a higher RMF prevalence ( $40 \% ; P=0.013$ ) compared to other moose herds surveyed; however, this is not entirely unexpected as previous reports have shown $R$. andersoni prevalence in Alaska as high as $70 \%$ [14]. Variations in vector and, or host density could potentially play an important

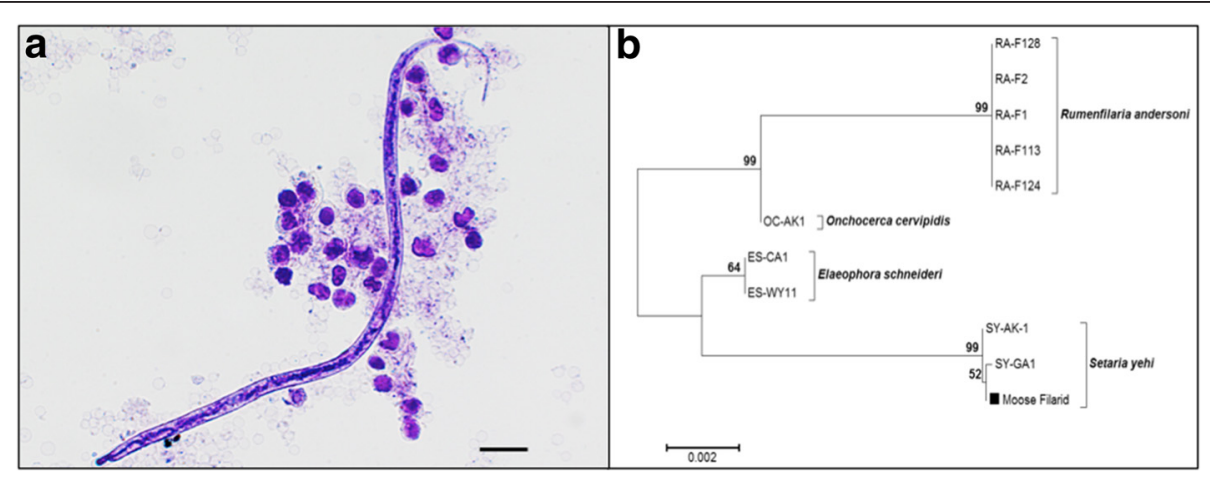

Fig. 3 Morphological and genetic characterization identifies moose filarioid as Setaria yehi. a Image of unidentified microfilaria observed in blood from Minnesota moose. Sample was stained with methylene blue and image taken under a bright light microscope at 200x magnification. Scale-bar: $20 \mu \mathrm{m}$. b Phylogenetic comparison of 18S rRNA sequences (796 base pairs) from unknown filarioid (black box) and other known filarioid parasites of ungulates, with history inferred using the Neighbor-Joining method and evolutionary distances computed using the Kimura 2-parameter method. Tree is drawn to scale. Bootstrap values (×1000) are shown above branches. GenBank accession numbers for all isolates are listed in Tables 1 and 3 
role in $R$. andersoni prevalence and may be contributing to this disparity. It is possible that $R$. andersoni and/or its preferred vector may be more highly adapted to subarctic climates, proliferating more easily in a colder environment. The recent rapid expansion of $R$. andersoni into the subarctic regions of Finland $[15,16]$ supports this hypothesis, but additional evidence is needed to substantiate this claim. Further research on moose densities, vector distribution and competency, and distribution of other possible definitive hosts of $R$. andersoni will be useful in understanding if the parasite is more adapted to subarctic climates or if it is strictly a host and/or vector densitydependent mechanism.

Surveys for RMF in other cervid species revealed $R$. andersoni occurs in white-tailed deer, and deer can serve as a definitive host for the filarioid (Fig. 1a). Presence of RMF in white-tailed deer was documented once before in 2005 while investigating the emergence of $R$. andersoni in Finnish semi-domesticated reindeer [16]. The authors observed $22 \%$ of the deer surveyed in Finland were RMF-positive, higher than the $5.6 \%$ prevalence value observed in deer from Minnesota. As mentioned above, it is possible the parasite is better adapted to subarctic environments, thus explaining the lower parasite prevalence at the more southern latitude; however, a larger sample size along a latitudinal gradient would be needed to properly address that particular hypothesis.

In addition to white-tailed deer, our survey included specimens from caribou, elk, and mule deer from various geographical locales. We were unable to find evidence of $R$. andersoni infections within any of these species. This was surprising, as $R$. andersoni was found to be a common and abundant parasite in reindeer of Finland, with prevalence as high as $90 \%$ in some locations [16]. Although the caribou and reindeer herds from Alaska and Finland are different subspecies, and it is possible that Alaskan caribou may be genetically more resistant to $R$. andersoni infection, we suspect the absence of $R$. andersoni in our Alaskan caribou specimens may be due to an insufficient sample size rather than a lack of host-parasite coadaptation. Additionally, the number of elk and mule deer surveyed may have been insufficient, resulting in no RMF detected in the modified Knott's test; however, it is also possible these species are not suitable hosts for the nematode. Further sampling will better characterize $R$. andersoni host range and suitability among North American cervid populations.

It should be noted that the use of the modified Knott's test to estimate prevalence based on finding microfilariae in blood samples could result in an underestimation of the true prevalence of $R$. andersoni in their mammalian hosts. Timing of sample collection could significantly influence estimated prevalence values, potentially causing early-stage infections to be missed or causing false negatives with the synchronous influx of microfilariae into general circulation that coincides with circadian rhythms [16]. Furthermore, the modified Knott's test is unable to detect infections with nematodes of a single sex due to the female filarioid being unable to mate and produce progeny. At this time, the only alternative method to estimate prevalence would be to grossly examine host carcasses for the presence of adult $R$. andersoni, presumably in the lymphatic vessels of the rumen; however, in addition to being a laborious and tedious process, fresh wildlife carcasses can be difficult to obtain.

To gain a better understanding of the diversity and genetic variation of $R$. andersoni nematodes, we compared ITS-1 sequences obtained from RMF-positive blood samples (Fig. 2). Our phylogenetic analysis suggests there are two clades of $R$. andersoni in North America, with all Montana isolates associating with Clade A and all Maine isolates falling into Clade B. Interestingly, Minnesota isolates have representatives in both clades. Possible reasons for the mixed clades in Minnesota include potential overlapping RMF populations or previous cervid translocation events. It is also possible multiple paratypes of $R$. andersoni circulate within the Minnesota cervid host populations. Previous morphological analyses of $R$. andersoni specimens revealed nematodes often differ by the number and arrangement of caudal papillae present $[13,15]$. Combined, our ITS-1 data and the morphological descriptions suggest at least two separate $R$. andersoni populations exist; however, a dual DNA-based and adult morphological study will be needed to identify if there is a distinct relationship between caudal papillae phenotypes and phylogenetic assortment. Furthermore, studies comparing $R$. andersoni genotypes in the vector and cervid hosts would provide additional insight into $R$. andersoni population dynamics and may help to identify factors driving $R$. andersoni transmission and maintenance within the environment.

Here, we also observed our $R$. andersoni reference nematode's (RA-F3) ITS-1 sequence clustered into Clade A with isolates from Minnesota and Montana (Fig. 2). This is significant as RA-F3 was isolated from a reindeer in Finland, which recently experienced a significant expansion of $R$. andersoni in wild and domestic reindeer herds [16]. Researchers hypothesized the colonization of Finnish cervids with $R$. andersoni resulted from the introduction of non-native white-tailed deer from North America, specifically the U.S. state of Minnesota. Five deer, one male and four females, were imported into southern Finland in 1935 as a gift from Finnish immigrants from northern Minnesota [26]. If Finnish $R$. andersoni nematodes' ancestors originated from Minnesota, we would expect the Finnish ITS-1 sequences to be similar to those of North American specimens, especially those of Minnesota; conversely, we would expect significant genetic variation if 
the Finnish population had an extended history of geographical distribution and isolation in Fennoscandia. Our data demonstrate a lack of divergence between these isolates, indicating that a more recent, anthropogenicallydriven introduction of the parasite occurred, supporting the Minnesota theory, rather than an introduction coinciding with the geographical colonization by moose from Central Europe and Russia after the last glaciation, approximately 10,000 years ago [27].

In addition to the presence of RMF, our data revealed another filarioid circulating within the Minnesota moose herd (Fig. 3). Sequencing and phylogenetics confirmed the identity of this filarial nematode to be S. yehi, a common parasite of cervids in North America (Fig. 3b). Adult S. yehi are most commonly found in the abdominal cavity and produce microfilariae that circulate within the bloodstream $[14,28]$. Various mosquito genera are considered to be the major vectors of this nematode [29]. Although not much is known about the distribution of $S$. yehi in moose, previous reports documented $S$. yehi-infected moose in Alaska [30], Alberta [31], Ontario [32], and Wisconsin [30], suggesting S. yehi is likely widespread amongst North American moose herds. This parasite can cause a wide range of disease in cervids. Mild fibrin formation on serosal surfaces has been reported in $S$. yehi infected white-tailed deer and chronic peritonitis has been reported in infected Alaskan reindeer [33]. Additionally, intense inflammation, secondary bacterial peritonitis and mortality in nine free-ranging moose calves were attributed to migrating $S$. yehi and high intensities of microfilariae [14]. However, to our knowledge there have been no reports of clinical infections in adult moose, and the incidence of morbidity and mortality associated with $S$. yehi infections within moose populations have not been studied. Thus, it is unknown the impact S. yehi infections may have on moose population dynamics.

Although other nematode parasites are known to contribute to moose morbidity and mortality [6], our study was unable to establish a clear association between prevalence of $R$. andersoni and declining populations of moose. No significant difference in RMF prevalence was observed between the Minnesota moose herd, which is exhibiting a severe population decline [4], and those of Montana and New Hampshire, which are also decreasing $[1,3]$, albeit less dramatically; Washington, which has steadily increased in numbers since 1922 [34]; or Maine, where the moose population has increased over the last decade [35, 36] (Fig. 1c). Thus, prevalence of $R$. andersoni does not appear to have an obvious association with declining moose populations. At this time, it is still unknown what impact, if any, $R$. andersoni infection may have on the health of the moose host. Laaksonen et al., in 2010, observed macroscopic inflammatory changes within the ruminal lymphatic vessels of infected Finnish reindeer [15]; however, there have yet to be any reports of pathological changes associated with moose. Given the high prevalence of RMF in moose and the high prevalence and intensity of RMF in Finnish reindeer, future studies on potential subclinical and clinical disease is warranted. It is reasonable to hypothesize that infection with $R$. andersoni has a metabolic cost, and it is possible that heavy worm burdens or systemic microfilaremia would result in adverse health effects, potentially rendering the host more susceptible to other infectious agents or poor body condition, but future studies will be required to assess the validity of these hypotheses.

\section{Conclusions}

Rumenfilaria andersoni is a nematode widespread throughout moose herds of North America. In addition to moose, white-tailed deer appears to be a natural, definitive host of this parasite. Recognizing the geographical distribution and host range of this filarioid is especially important for preventing the introduction of $R$. andersoni into naïve populations by translocation of animals by state conservation agencies or commercial hunting businesses. Our genetic comparison of $R$. andersoni isolates supports the hypothesis that the recent and rapid spread of $R$. andersoni in Finnish reindeer was due to the introduction of white-tailed deer from North America [15, 16], and further underscores the importance of a better awareness of $R$. andersoni biology. We were unable to correlate levels of parasite prevalence with moose population decline, and it is still unknown if $R$. andersoni infection can lead to clinical or subclinical disease. Continued efforts to document this parasite in cervid hosts will help to provide clarity on this topic.

\section{Abbreviations}

Cl, confidence interval; ITS-1, first internal transcribed spacer region of the rRNA gene; RMF, Rumenfilaria andersoni microfilariae

\footnotetext{
Acknowledgements

Reference nematodes were generously donated by Kimberlee Beckmen at the Alaskan Department of Wildlife, Fish and Game and John Henningsen at the Wyoming State Veterinary Laboratory. The authors also thank Nick DeCesare of the Montana Fish and Wildlife Department, Ella Rowan and Kristin Mansfield of the Washington State Department of Fish and Wildlife, Lee Kantar of Maine Department of Inland Fisheries and Wildlife, Anne Lichtenwalner of the University of Maine, and Henry Jones of the University of New Hampshire for providing cervid blood samples for analysis. We thank Ann Reed at the University of Tennessee Institute of Agriculture for statistical consultation. We also thank Roger Moon of the Department of Entomology at the University of Minnesota, for his helpful comments while preparing this manuscript, and Misty Bailey (funded by the University of Tennessee College of Veterinary Medicine) for technical editing of the manuscript. This work was partially supported by the Minnesota Department of Natural Resources Section of Wildlife and the University of Tennessee Department of Biomedical and Diagnostic Sciences. Finnish samples were collected as part of the project "Reindeer health in the changing environment," funded by the Finnish Ministry of Agriculture and Forestry (MAKERA) and the EurgNegVec COST Action TD1303. Fellowship funding for CG was provided by the University of Tennessee Department of Microbiology.
} 


\section{Funding}

This work was partially supported by the Minnesota Department of Natural Resources Section of Wildlife and the University of Tennessee Department of Biomedical and Diagnostic Sciences. Finnish samples were collected as part of the project "Reindeer health in the changing environment," funded by the Finnish Ministry of Agriculture and Forestry (MAKERA) and the EurgNegVec COST Action TD1303. Fellowship funding for graduate student (CG) was provided by the University of Tennessee Department of Microbiology.

\section{Availability of data and materials}

The datasets supporting the conclusions of this article have been included within the article. Genetic sequence data has been submitted to the GenBank (National Center for Biotechnology Information) database under accession numbers KT020850, KT031392-KT031393; KT873719-KT873733; KT878970-KT878979l; and KU757075-KU757077.

\section{Authors' contributions}

CG and RG conceived and designed the study and drafted the manuscript. CG and JE performed the modified Knott's tests and molecular analysis of reference nematodes. CG completed all genetic analysis of RMF isolates. MC and $\mathrm{EH}$ orchestrated and oversaw collection of Minnesota cervid samples and drafting of the manuscript. AO and SL collected Finnish samples and were active in writing the manuscript. All authors were involved in analyses of data, and reviewed and approved the final manuscript

\section{Competing interests}

The authors declare that they have no competing interests.

\section{Consent for publication}

Not applicable.

\section{Ethics approval and consent to participate}

All protocols for the handling and collection of materials from animals followed the guidelines set by the American Society of Mammalogists [37, 38] and the American Veterinary Medical Association [39].

\section{Author details}

'Department of Microbiology, University of Tennessee, M409 Walters Life Sciences, Knoxville, TN 37996-0845, USA. ${ }^{2}$ Minnesota Department of Natural Resources, 500 Lafayette Road, St. Paul, MN 55155-4040, USA. ${ }^{3}$ Department of Veterinary Biosciences, University of Helsinki, Fabianinkatu 33, Fl-00014 Helsinki, Finland. ${ }^{4}$ Finnish Food Safety Authority Evira, Production Animal and Wildlife Health Research Unit (FINPAR), Elektroniikkatie 3, FI-90590 Oulu, Finland. ${ }^{5}$ Department of Biomedical and Diagnostic Sciences, College of Veterinary Medicine, University of Tennessee, 2407 River Drive, Knoxville, TN 37996, USA.

\section{Received: 30 March 2016 Accepted: 3 August 2016}

\section{Published online: 12 August 2016}

\section{References}

1. DeCesare NJ, Smucker TD, Garrott RA, Gude JA. Moose management in Montana. Alces. 2014;50:35-51.

2. Monteith KL, Klaver RW, Hersey KR, Holland AA, Thomas TP, Kauffman MJ. Effects of climate and plant phenology on recruitment of moose at the southern extent of their range. Oecologia. 2015;178:1137-48.

3. Rines KM. New Hampshire moose assessment 2015. Concord: New Hampshire Department of Fish and Game; 2015.

4. Del Giudice GD. Aerial moose survey. St. Paul: Minnesota Department of Natural Resources; 2015.

5. Murray DL, Cox EW, Ballard WB, Whitlaw HA, Lenarz MS, Custer TW, et al, Pathogens, nutritional deficiency, and climate influences on a declining moose population. Wildl Monogr. 2006;166:1-29.

6. Wunschmann A, Armien AG, Butler E, Schrage M, Stromberg B, Bender JB, et al. Necropsy findings in 62 opportunistically collected free-ranging moose (Alces alces) from Minnesota, USA (2003-2013). J Wildl Dis. 2015:51:157-65.

7. Mech LD, Fienberg J. Re-evaluating the northeastern Minnesota moose decline and the role of wolves. J Wildl Manag. 2014;78:1143-50.

8. Carstensen M, Hildebrand EC, Plattner D, Dexter MH, Jennelle C, Wright RG. Determining cause-specific mortality of adult moose in northeast Minnesota. In: Cornicelli M, Carstensen M, Grund M, Larsen M, Lawrence J, editors. Summaries of wildlife research findings. St. Paul: Minnesota Department of Natural Resources; 2015. p. 161-71.

9. Lenarz MS, Nelson ME, Schrage MW, Edwards AJ. Temperature mediated moose survival in Northeastern Minnesota. J Wildl Manag. 2009;73(4):503-10.

10. Madden DJ, Spraker TR, Adrian WJ. Elaeophora schneideri in moose (Alces alces) from Colorado. J Wildl Dis. 1991;27:340-1.

11. Smith HJ, Archibald RM. Moose sickness, a neurological disease of moose infected with the common cervine parasite, Elaphostrongylus tenuis. Can Vet J. 1967;8:173-7.

12. Laaksonen S, Kuusela J, Nikander S, Nylund M, Oksanen A. Outbreak of parasitic peritonitis in reindeer in Finland. Vet Rec. 2007;160:835-41.

13. Lankester M, Snider JB. Rumenfilaria andersoni n. gen., n. sp. (Nematoda: Filarioidea) in moose from northwestern Ontario, Canada. Can J Zool. 1982;60:2455-8.

14. Kutz SJ, Ducrocq J, Verocai GG, Hoar BM, Colwell DD, Beckmen KB, et al Parasites in ungulates of Arctic North America and Greenland: a view of contemporary diversity, ecology, and impact in a world under change. Adv Parasitol. 2012;79:164-5.

15. Laaksonen S, Saari S, Nikander S, Oksanen A, Bain O. Lymphatic dwelling filarioid nematodes in reindeer Rangifer tarandus tarandus, (Cervidae) in Finland, identified as Rumenfilaria andersoni Lankester \& Snider, 1982. (Nematoda: Onchocercidae: Splendidofilariinae). Parasite. 2010;17:23.

16. Laaksonen S, Oksanen A, Hoberg E. A lymphatic dwelling filarioid nematode Rumenfilaria andersoni (Filarioidea; Splendidofilariinae), is an emerging parasite in Finnish cervids. Parasit Vectors. 2015;8:282.

17. Adcock JL, Hibler CP. Vascular and neuro-opthalmic pathology of elaeophorosis in elk. Pathol Vet. 1969;6:185-213.

18. Carrasco L, Fierro Y, Sanchez-Castillejo JM, Bautista MJ, Gomez-Villamandos JC, Sierra MA. Elaeophorosis in red deer caused by Elaeophora elaphi: Lesions of natural disease. Vet Pathol. 1995;32:250-7.

19. Bennuru S, Nutman TB. Lymphangiogenesis and lymphatic remodeling induced by filarial parasites: implications for pathogenesis. PLoS Pathog. 2009;5(12):e1000688

20. Georgi JR. Diagnostic parasitology. In: Georgi JR, Georgi ME, editors. Georgi's parasitology for veterinarians. 4th ed. Philadelphia: W.B. Saunders Company; 1985. p. 261-2.

21. Anderson RC. The superfamily filarioidea. In: Nematode parasites of vertebrates: their development and transmission. 2nd ed. New York: CABI Publishing; 2000. p. 467-532.

22. Nuchprayoon S, Junpee A, Poovorawan Y, Scott AL. Detection and differentiation of filarial parasites by universal primers and polymerase chain reaction-restriction fragment length polymorphism analysis. Am J Trop Med Hyg. 2005;73:895-900

23. Tamura K, Stecher G, Peterson D, Filipski A, Kumar S. MEGA6: Molecular Evolutionary Genetics Analysis version 6.0. Mol Biol Evol. 2013;30:2725-9.

24. Floyd RM, Rogers AD, Lambshead PJD, Smith CR. Nematode-specific PCR primers for the $18 \mathrm{~S}$ small subunit rRNA gene. Mol Ecol Notes. 2005;5:611-2

25. Prestwood AK, Pursglove SR. Prevalence and distribution of Setaria yehi in southeastern white-tailed deer. J Am Vet Med Assoc. 1977;171:933-5.

26. Ruusila V, Kojola I. Ungulates and their management in Finland. In: Apollonio M, Andersen R, Putman R, editors. European ungulates and their management in the 21st Century. 1st ed. New York: Cambridge University Press; 2010. p. 86-102.

27. Niedzialkowska M, Hundertmark KJ, Jedrzejewska B, Niedzialkowski K, Sidorovich VE, Górny M, et al. Spatial structure in European moose (Alces alces) reveal a complex population history. J Biogeogr. 2014;11:2173-84.

28. Cook TW, Ridgeway BT, Andrews R, Hodge J. Gastro-intestinal helminths in white-tailed deer (Odocoileus virginianus) of Illinois. J Wildl Dis. 1979;15:405-8.

29. Allan S. Biting flies (Class Insecta: Order Diptera). In: Samuel WM, Pybus MJ, Kocan AA, editors. Parasitic diseases of wild mammals. 2nd ed. Ames: lowa State University Press; 2001. p. 18-45.

30. Becklund W, Walker M. Taxonomy, hosts, and geographical distribution of Setaria (Nematoda: Filarioidea) in the United States and Canada. J Parasitol. 1969:55:359-68.

31. Samuel WM, Barrett MW, Lynch GM. Helminths in moose of Alberta. Can J Zool. 1976:54:307-12.

32. Hoeve J, Joachim DG, Addison EM. Parasites of moose (Alces alces) from an agricultural area of eastern Ontario. J Wildl Dis. 1988;24:371-4.

33. Dieterich RA, Luick JR. The occurence of Setaria in reindeer. J Wildl Dis. 1971;7:242-5 
34. Harris R, Hansen S, Oyster J, Mansfield K, Rowan E, Goerz J, et al. Moose abundance, distribution, and demographic characteristics in eastern Washington. Olympia: Washington Department of Fish and Wildlife; 2015.

35. Morris KI. Moose assessment. Augusta: Maine Department of Inland Fisheries and Wildlife; 1999.

36. Woodcock CE, Augusta ME, editors. Research and management report. Maine: Department of Inland Fisheries and Wildlife; 2014.

37. American Society of Mammalogists. Guidelines for the capture, handling, and care of mammals as approved by the American Society of Mammalogists. J Mammalogy. 1998;79:1416-31.

38. Sikes RS, Gannon WL. Animal Care and Use Committee of the American Society of Mammalogists. Guidelines of the American Society of Mammalogist for the use of wild mammals in research. J Mammalogy. 2011;92:235-53.

39. American Veterinary Medical Association Panel on Euthanasia (AVMA). AVMA Guidelines for the euthanasia of animals. 2013. https://www.avma. org/KB/Policies/Documents/euthanasia.pdf. Accessed 20 Jun 2013.

Submit your next manuscript to BioMed Central and we will help you at every step:

- We accept pre-submission inquiries

- Our selector tool helps you to find the most relevant journal

- We provide round the clock customer support

- Convenient online submission

- Thorough peer review

- Inclusion in PubMed and all major indexing services

- Maximum visibility for your research

Submit your manuscript at www.biomedcentral.com/submit
Biomed Central 